\title{
Effect of Copper pretreatment on optical and electrical properties of camphor based graphene by chemical vapour deposition
}

Harsh Chaliyawala ( $\sim$ harsh.physics7@gmail.com )

Sardar Vallabhbhai National Institute of Technology https://orcid.org/0000-0002-5418-3223

R. Narasimman

Vikram Sarabhai Space Centre

Ranjan K. Pati

Pandit Deendayal Petroleum University

Indrajit Mukhopadhyay

Pandit Deendayal Petroleum University

Abhijit Ray

Pandit Deendayal Petroleum University

\section{Research Article}

Keywords: Graphene, Camphor, Copper foil, CVD, Schottky diode.

Posted Date: June 1st, 2021

DOI: https://doi.org/10.21203/rs.3.rs-281338/v1

License: (c) (i) This work is licensed under a Creative Commons Attribution 4.0 International License.

Read Full License

Version of Record: A version of this preprint was published at Journal of Materials Science: Materials in Electronics on June 4th, 2021. See the published version at https://doi.org/10.1007/s10854-021-06300-y. 


\section{Abstract}

Polycrystalline copper $(\mathrm{Cu})$ foil is widely used as catalytic substrate for graphene growth in chemical vapor deposition (CVD) technique. The surface properties of the Cu foil strongly affect the growth behavior and final quality of CVD-grown graphene. The effect of pretreatment of Cu foil using four different solutions (acetone, acetic acid, $\mathrm{HCl}$ and $\mathrm{HNO}_{3}$ ) on the graphene growth held in atmospheric pressure CVD and its subsequent impact on electrical and optical properties are investigated. Natural camphor is used as the solid carbon precursor. The surface characteristics before and after the growth are studied using scanning electron microscopy and atomic force microscopy. The pretreatment conditions of $\mathrm{Cu}$ and the growth of graphene from camphor were correlated using Raman spectroscopy, optical and electrical characteristics. Our findings suggest that $\mathrm{HCl}$ pretreated $\mathrm{Cu}$ foil exhibited largedomain, uniform coverage of the transferred graphene with excellent optical (>93\% at $550 \mathrm{~nm}$ ) and electrical properties (sheet resistance of $861 \pm 40 \mathrm{ohm} / \mathrm{sq}$ ), with promisingly low RMS value of roughness $(38 \mathrm{~nm})$. The pretreatment process improved the quality of graphene by removing the surface impurity particles and surface native oxides. A Schottky junction diode of graphene/n-silicon is fabricated by transferring the graphene to $\mathrm{SiO}_{2} / \mathrm{Si}$ substrate under dark and illuminated conditions is also demonstrated to establish its potential in micro- and opto-electronics.

\section{Introduction}

Single-Layer Graphene (SLG), a one-atom-thick sheet of hexagonally arrayed $\mathrm{sp}^{2}$-bonded carbon atoms, has received much attention due to its interesting electrical, mechanical, optical, and thermal properties [1-6]. Electronic and optoelectronic applications such as transparent conducting electrode, solar cells, photodetectors and field-effect transistors, demand graphene film with very high quality and large domain size in a larger area $[1,7,8]$. The graphene can be produced by various methods such as micromechanical cleavage [1], chemical vapor deposition (CVD) [9, 10], epitaxial growth on Silicon Carbide (SiC) [11] and reduction of graphene oxide [12, 13]. Among these techniques, CVD method is emerged as a simple, scalable and straight forward for high-quality, large-area graphene. In this technique, the carbon precursors are decomposed on the catalytic metal surface such as $\mathrm{Cu}, \mathrm{Ni}$, Pt, Ir, etc $[9,10,14,15]$. The resulting graphene on metal substrate can be easily transferred to any desirable substrates by employing simple transferring process without affecting much of the properties of the graphene. Gaseous hydrocarbon like $\mathrm{CH}_{4}, \mathrm{C}_{2} \mathrm{H}_{4}$, or $\mathrm{C}_{2} \mathrm{H}_{2}$ is introduced to a chamber and dissociated on metal surfaces ( $\mathrm{Cu}, \mathrm{Pt}$ or $\mathrm{Ni}$ ) at elevated temperature to form active carbon species, which constitute the long chain of graphene $[10,16,17]$. Moreover, liquid carbon sources, like benzene, toluene, methanol, ethanol, propanol and pentane have been used for high-quality graphene growth $[18,19]$. Recently, solid carbon source such as camphor has taken much attraction for the synthesis to form a large area monolayer graphene by very facile and novel approach [20-23]. Kalita et al. has performed the growth of mono/bi- layer graphene by employing the camphor as a carbon source on different metal substrates [20-24]. 
Polycrystalline Cu foils is most widely used catalyst in CVD technique to grow monolayer graphene due to its low-cost, scalability, and compatibility with roll-to-roll processing $[25,26]$. The electrical and physical properties of CVD grown graphene sheets are limited by polycrystalline nature and smaller grain size of $\mathrm{Cu}$ foil. It is well-known that $\mathrm{Cu}$ is having low carbon solubility and thus the graphene growth is surfacecatalyzed process which limits to monolayer graphene [27, 28]. We understood that the surface properties of $\mathrm{Cu}$ foil are very important as it strongly influence the growth of graphene [29,30]. In particular, the graphene growth can be obstructing for instance due to $\mathrm{Cu}$ surface roughness, contamination, crystallographic orientations and grain size. These properties can influence the nucleation density and growth rate of the graphene or control the alignment of graphene domains $[10,26,31]$. As received $\mathrm{Cu}$ foils are known to have surface contamination particles, striation due to the cold- rolling process during manufacturing and native oxide on the surface. Also, some of the Cu foils are supplied with oxidative protective coating. These impurities and surface defects are reported to have significant effect on the final properties of graphene by acting as hetero-nucleation centers. The Cu foil pretreatment is prerequisite for preparing high-quality graphene by CVD technique [32-41]. Different pretreatment procedures for $\mathrm{Cu}$ foils are reported; among them (1) acetic acid $\left(\mathrm{CH}_{3} \mathrm{COOH}\right)$ [42], (2) dilute $\mathrm{HCl}$ [37] (3) dilute $\mathrm{HNO}_{3}$ [32], (4) ferric chloride [34], (5) $\mathrm{KOH}$ [36], (6) potassium peroxodisulfide [33] and (7) electropolishing [43] are explored.

Despite of significant efforts made in recent years, the growth of high-quality CVD graphene from camphor and its applications are not addressed with respect to the pretreatment effect on Cu foil surfaces. In the present work, we have critically addressed a systematic analysis of the surface roughness and morphology of the pretreated $\mathrm{Cu}$ foil for the growth of graphene from camphor. Atmospheric pressure CVD (APCVD) is used to grow the graphene and corroborated the optical and electrical properties with different pretreated $\mathrm{Cu}$ foil in detail. A graphene/n-Si schottky junction photodiode by applying the optimally grown graphene is also fabricated in above mentioned scheme and demonstrated its device potential.

\section{Experimental Section}

\subsection{Pretreatment of copper foil}

The $25 \mu \mathrm{m}$ copper foil (13382) from Alfa Aaser, USA was used as a metal substrate for graphene growth. The Cu foil was cut into the dimensions of $2.5 \mathrm{~cm} \times 2.5 \mathrm{~cm}$. Then, they were cleaned by different cleaning procedures. The detailed pretreatment procedures for the $\mathrm{Cu}$ foils are given as follows:

(1) The Cu foil was ultrasonically cleaned in acetone for 10 minutes.

(2) Cu foil was cleaned with acetone, DI water and then immersed in glacial acetic acid at room temperature for 10 minutes. After that the $\mathrm{Cu}$ foil was rinsed with DI water three times and finally washed with isopropanol (IPA). 
(3) Cu foil was cleaned with acetone, DI water and then immersed in 2:1 ratio of DI water: $\mathrm{HCl}$ at room temperature for 5 minutes. Hereafter, the Cu foil was rinsed with DI water three times and finally washed with isopropanol (IPA).

(4) Cu foil was cleaned with acetone, DI water and then immersed in 5 wt.\% nitric acid at room temperature for 30 seconds. Finally, Cu foil was rinsed with DI water three times and washed with isopropanol (IPA). At the final stage, Cu foils were dried under the stream of Ar gas before loading into the furnace.

\subsection{Synthesis of graphene by atmospheric pressure chemical vapor deposition (APCVD)}

A quartz tube with a diameter of $55 \mathrm{~mm}$ and length of $1250 \mathrm{~mm}$ was placed in a horizontal split furnace. The pretreated $\mathrm{Cu}$ foils were loaded into the quartz tube and placed in the high temperature zone. The $\mathrm{Cu}$ foil was kept $\sim 10^{\circ}$ of inclination position, facing towards the gas flow. The camphor was placed in a quartz boat and kept at an optimized distance from the heating zone in order to avoid the unwanted sublimation during pre-annealing and annealing step. The Cu foil was heated from room temperature to $1020^{\circ} \mathrm{C}$ at the heating rate of $\sim 15^{\circ} \mathrm{C} / \mathrm{min}$ under the flow of $200 \mathrm{sccm}$ high purity Ar flow followed by annealing for 15 minutes under the flow of $100 \mathrm{sccm} \mathrm{H}$. For growth of graphene, the gas composition was changed to $\mathrm{Ar}: \mathrm{H}_{2}$ in $98: 2$ ratio. The camphor was evaporated at the heating rate of $10^{\circ} \mathrm{C} / \mathrm{min}$ to $200^{\circ} \mathrm{C}$. The total growth time was nearly $10-15 \mathrm{~min}$. After the growth, the operation was switched off and chamber was allowed to cool naturally under the flow of $\mathrm{Ar}$ and $\mathrm{H}_{2}$ gas.

\subsection{Transfer of graphene to $\mathrm{SiO}_{2} / \mathrm{Si}$ and glass substrates}

The graphene covered Cu was coated with PMMA by spin coating at $3000 \mathrm{rpm}$ for 60 seconds and 1000 rpm for 60 seconds, followed by baking of PMMA/graphene/copper at $180^{\circ} \mathrm{C}$ for 2 minutes. Then the copper was etched by placing the samples over $0.5 \mathrm{M}\left(\mathrm{NH}_{4}\right)_{2} \mathrm{~S}_{2} \mathrm{O}_{8}$ for 6 hours. The graphene grown on the back side of the Cu was removed by washing under the stream of DI water and placed again on the etching solution. The PMMA/graphene was repeatedly cleaned in DI water for 5 to 6 times. Then the $\mathrm{PMMA} /$ graphene was transferred on to the glass or $\mathrm{SiO}_{2} / \mathrm{Si}$ substrates. The substrates were kept overnight for the complete adhesion to the substrate. Finally, the PMMA layer was removed by dipping in acetone for 30 minutes and after that repeatedly washed with DI water and IPA.

\subsection{Characterization}

FE-SEM analysis was carried out using a SEM microscope (Zeiss, Ultra-55) using $5.0 \mathrm{kV}$ acceleration voltage. The elemental composition of the pretreated Cu foils was investigated by using X-ray photoelectron spectroscope (XPS, PHI5000 VersaProbe II, ULVAC-PHI, INC, USA) with a monochromated Al-Ka X-ray source operating at $14 \mathrm{kV}$ and $220 \mathrm{~W}$. The XPS data were processed using PHI's Multipak software. The binding energy was referenced to the $\mathrm{C} 1 \mathrm{~s}$ peak at $284.8 \mathrm{eV}$. The sheet resistance of all samples was measured using a four-point resistance meter with silver paste as the contact pad. Optical transmittance of graphene films was measured using a UV-Vis spectrophotometer (UV-2600, Shimadzu). Raman measurements were carried out to study the identification of graphene by using a $532 \mathrm{~nm}$ laser 
source with a Micro Raman microscope (inVia, Renishaw, UK). The measurements were carried out on graphene transferred on to $\mathrm{SiO}_{2} / \mathrm{Si}$ substrate. The laser power used was $2.5 \mathrm{~mW}$, with accumulation of 30 sec.

\subsection{Graphene/n-silicon schottky junction diode}

In order to establish the device potential of the graphene, simple schottky diode was fabricated using the graphene as metal with $n$-type silicon as the semiconductor counterpart. The device was fabricated as follows. A $150 \mathrm{~nm} \mathrm{SiO}$ was coated on $\mathrm{n}-\mathrm{Si}(100)(1 \mathrm{~cm} \times 1 \mathrm{~cm}$ pieces) by thermally annealing the substrates at $1000^{\circ} \mathrm{C}$ for $4 \mathrm{hr}$ under $\mathrm{O}_{2}$ atmosphere. The buffer oxide etch solution (BOE, NH${ }_{4} \mathrm{~F} / \mathrm{HF} ; 6: 1$ ) was used for $\mathrm{SiO}_{2}$ etching and to expose the $\mathrm{n}$-Si with a window size of $0.3 \mathrm{~cm} \times 0.3 \mathrm{~cm}\left(\sim 0.09 \mathrm{~cm}^{2}\right)$. The wafers were then repeatedly cleaned with DI water. Immediately, PMMA-coated graphene was transferred on to the $\mathrm{SiO}_{2}$ coated $\mathrm{n}$-Si on to the active area window. The PMMA was removed by acetone. The contacts were made using silver paste on front side connected with $\mathrm{SiO}_{2}$ /graphene and similarly on the back side of the n-Si. In this configuration, graphene sheet serves as the metal semi-transparent upper electrode and the antireflection layer for schottky junction. The dark and light behavior of the schottky diode was tested under the solar simulator integrated with a source-measure unit (Agilent) by applying the bias of -5 to $+5 \mathrm{~V}$.

\section{Results And Discussion}

\subsection{Effect of pretreatment on the Cu foil}

The quality of graphene strongly depends on the nucleation process which gets affected by the surface properties of $\mathrm{Cu}$ foils $[36,39,43]$. Thus, the $\mathrm{Cu}$ foil cleaning process is very important to get reproducible and high-quality large domain graphene because the as received $\mathrm{Cu}$ foils usually contains contamination particles, native oxides, protective coating, striation lines and surface defects $[35,39]$. The SEM images of the acetone, $\mathrm{AA}, \mathrm{HCl}$ and $\mathrm{HNO}_{3}$ pretreated $\mathrm{Cu}$ foils are shown in Fig. $1(\mathbf{a}-\mathbf{d})$. The acetone treated foil contains surface inhomogeneity and striation lines which are usually formed during the rolling of the $\mathrm{Cu}$ foils. On the other hand, $\mathrm{AA}, \mathrm{HCl}$ and $\mathrm{HNO}_{3}$ pretreated $\mathrm{Cu}$ foils have shown a reduction in the surface inhomogeneity and impurity particles. Among these pretreatment conditions, $\mathrm{HCl}$ treated $\mathrm{Cu}$ foils exhibited smooth surface. Acetone and IPA cleaning is not sufficient for the removal of the particles, even though this step is important in order to get rid of the organic containments on the Cu foils. The particles are partially removed from the $\mathrm{Cu}$ foil when it is treated with AA. However, complete removal of the particles is required to get the high quality graphene. It is seen that, $\mathrm{HCl}$ and $\mathrm{HNO}_{3}$ pretreatments remove the particle contamination by more than $95 \%$ from the Cu foil. Further we have carried out AFM analysis to understand the Cu topology after different pretreatment conditions (Fig. $1(\mathbf{e}-\mathbf{h})$ ). The influence of the pretreatment process with different pretreatment conditions can be seen from the RMS values calculated from the AFM analysis. The RMS values of the roughness estimated from the AFM analysis for the acetone pretreated $\mathrm{Cu}$ foil was found to be $96 \mathrm{~nm}$. 
The acetone is expected to remove any organic contaminants on the Cu foil but it does not reduce the protective coating, striation lines and oxide layers. The calculated RMS values of the $\mathrm{Cu}$ foils pretreated with $\mathrm{AA}, \mathrm{HCl}$ and $\mathrm{HNO}_{3}$ was found to reduce to 60,38 and $45 \mathrm{~nm}$, respectively. Moreover, the acid pretreatment process reduces the striation lines and removes the surface oxide layers. The $\mathrm{HCl}$ pretreatment reduced the RMS value to $38 \mathrm{~nm}$. A slight increase in the RMS value in the $\mathrm{HNO}_{3}$ treated Cufoil was observed which was due to the formation of pits and it was confirmed from the high magnification SEM images. This clearly shows the reduction in the RMS values because of the treatment process which will modify the graphene nucleation process.

In order to identify the effect of pretreatment on Cu foils, we carried out XPS analysis on all pretreated samples. Figure 2(a) shows the XPS survey spectra of the all samples which reveal the presence of $\mathrm{Cu}, \mathrm{C}$ and $\mathrm{O}$. Apart from these elements, we can clearly see that the acetone cleaned $\mathrm{Cu}$ foils contains various other elements such as $\mathrm{P}, \mathrm{Ca}, \mathrm{Mg}, \mathrm{Fe}, \mathrm{Si}$, etc. In general, when compared to the acetone treated $\mathrm{Cu}$ foils, other acid treatments resulted in the reduction of the impurities in the Cu foils. Importantly, we observed that the $\mathrm{Cu}$ foil pretreated with $\mathrm{HCl}$ resulted in the removal of the almost all the impurity particles. Figure 2(b) shows the magnified region between 150 and $60 \mathrm{eV}$ from the Fig. 2(a), which reveals the treatment effectively removed the $\mathrm{Si}$ and $\mathrm{P}$. This study confirms the effectiveness of the pretreatment protocols that has been adopted for the complete removal of impurities from the Cu foils. Especially, $\mathrm{HCl}$ pretreatment leads to the best results by reducing the surface impurities, thereby, decreasing the nucleation sites which helps in the growth of the uniform graphene over the Cu foil.

\subsection{APCVD Graphene growth}

Camphor is a botanical origin, low-cost, solid carbon precursors with the molecular structure consisting of hexagonal and pentagonal rings fused together. When camphor is evaporated slowly around $200^{\circ} \mathrm{C}$ and pushed to the growth region near the Cu substrate, the camphor molecules get dissociated to the smaller carbon fragments and deposited on the Cu substrate. Subsequently, the carbon nucleates and starts to grow from the further supply from the carbon precursor into 2D graphene. The camphor molecules decomposed on the surface of the $\mathrm{Cu}$ foil and the active carbon species forms the nucleation and further supply leads to the uniform growth of the graphene. The pretreated $\mathrm{Cu}$ foils were loaded inside the quartz tube furnace and graphene was grown as per the procedure mentioned in the experimental section. Figure 3 shows the low and high magnification SEM images of the graphene coated Cu foils at different pretreatment conditions. Low magnification images of $\mathrm{Cu}$ foils in all pretreatments show the rolling lines even after annealing and graphene growth. $\mathrm{HCl}$ pretreated $\mathrm{Cu}$ foil showed large graphene domains when compared to other pretreated Cu foils. This clearly revealed that the nucleation density of $\mathrm{HCl}$ pretreated $\mathrm{Cu}$ foil is reduced and thus leads to the growth of larger graphene domain. The high magnification image of graphene grown on acetone pretreated Cu foil, as displayed in Fig. 3 (a) and (e). The graphene does not grow uniformly on the $\mathrm{Cu}$ foil after acetone pretreatment. Figure $3(\mathrm{f}-\mathrm{h})$ shows graphene grown on AA, $\mathrm{HCl}$ and $\mathrm{HNO}_{3}$ pretreated $\mathrm{Cu}$ foils where we can see the reduction in impurity particles. However, $\mathrm{HCl}$ pretreated graphene/Cu foil showed reduced roughness and uniform coverage of the graphene. AA and 
$\mathrm{HNO}_{3}$ pretreated graphene/Cu foils showed rougher surfaces which is due toan increase in the nucleation density on the Cu foils.

In order to observe the coverage of graphene on the $\mathrm{Cu}$ foils with respect to the pre-treatment conditions, we oxidized the graphene/ Cu foils for 10 mins at $200^{\circ} \mathrm{C}$ in a muffle furnace. The direct optical observation on the evidence of graphene coverage is possible in this approach as the graphene coated $\mathrm{Cu}$ foil remains protected whereas the exposed $\mathrm{Cu}$ foil get oxidized and displays reddish color. Figure 4 shows the digital photograph of oxidized graphene on different pretreated $\mathrm{Cu}$ foils. We can see clearly the difference in the graphene coverage with respect to the $\mathrm{Cu}$ pretreatment. Acetone and $\mathrm{AA}$ pretreated $\mathrm{Cu}$ foils show non-uniform graphene coverage. The $\mathrm{HCl}$ pretreated $\mathrm{Cu}$ foil shows excellent protection towards the oxidation whereas $\mathrm{HNO}_{3}$ pretreated sample shows small grains and incomplete protection. Figure 5 shows the SEM micrographs of the oxidized graphene/Cu foils. The SEM images clearly reveal the oxidation of the unprotected $\mathrm{Cu}$ foil because of the incomplete coverage of the graphene layer. From these experiments, we can conclude that the samples treated with $\mathrm{HCl}$ shows complete uniform coverage of graphene. However, acetone and AA samples showed a very poor uniform coverage on the Cu foil.

\subsection{Properties of transferred graphene}

The APCVD graphene were transferred by PMMA-assisted technique to the glass and $150 \mathrm{~nm} \mathrm{SiO} / \mathrm{Si}$ substrate. The SEM micrographs of the graphene on $\mathrm{SiO}_{2} / \mathrm{Si}$ substrate are shown in Figure S1. Raman spectroscopy is a very useful technique to study the quality of the CVD-graphene [44, 45]. It provides the information on number of layers and defect structure in the CVD-graphene. Figure 6(a) shows the Raman spectra of the graphene transferred to $\mathrm{SiO}_{2} / \mathrm{Si}$ substrate grown at various pretreated Cu foils. The Raman spectrum of the graphene exhibits peaks at $\sim 2700 \mathrm{~cm}^{-1}$ (2D peak), $1580 \mathrm{~cm}^{-1}$ (G peak) and 1350 $\mathrm{cm}^{-1}$ (D peak) for all the variations, respectively. The intensity ratios of $2 \mathrm{D}$ to $\mathrm{G}\left(\mathrm{I}_{2 \mathrm{D}} / \mathrm{I}_{\mathrm{G}}\right)$ and $\mathrm{D}$ to $\mathrm{G}\left(\mathrm{I}_{\mathrm{D}} / \mathrm{I}_{\mathrm{G}}\right)$ provide information on the number of graphene layers and defect structure, respectively as graphically described in Fig. 6(b). The value of $\mathrm{I}_{2 \mathrm{D}} / \mathrm{I}_{\mathrm{G}}$ greater than 1 generally shows the single-layer graphene, value around 1 is bi-layer and value less than 1 shows the multi-layer in the CVD-graphene [44]. Also, increase in the $I_{D} / I_{G}$ ratio shows the increase in the defect in the graphene layer. In our case, an optimum formation of mono-bi layer graphene with almost no defect states of D peak is observed only in $\mathrm{HCl}$ treated $\mathrm{Cu}$ foil. It is to be noted that, Raman analysis has been carried out at various regions of the as grown graphene coated substrates.

The UV-visible transmittance and sheet resistance have been studied to understand the effect of Cu foil pretreatment conditions on the graphene on its optical and electrical properties, respectively. Figure 7 (a) and (b) shows the UV- visible transmittance spectra and sheet resistance of the graphene grown on the $\mathrm{Cu}$ foils pretreated at different conditions. The effect of the Cu pretreatment is having a strong influence on the transmittance and electrical properties of the graphene. The acetone and AA pretreated Cu foil derived graphene show low transmittance which may be due to the formation of the multilayer on the Cu foils. We have observed that the graphene grown on $\mathrm{HCl}$ and $\mathrm{HNO}_{3}$ pretreated $\mathrm{Cu}$ foils show high transmittance. The maximum transmittance achieved on glass at $550 \mathrm{~nm}$, for Acetone, $\mathrm{AA}, \mathrm{HCl}$ and $\mathrm{HNO}_{3}$ 
are $84.6,80.4,91.48$ and $89.4 \%$, respectively (inset of Fig. 7). Moreover, the sheet resistance of the graphene from the acetone and AA pretreated Cu foils shows $1571 \pm 37$ and $1197 \pm 23 \Omega$ /sq, respectively. The $\mathrm{HCl}$ and $\mathrm{HNO}_{3}$ samples show the value of $861 \pm 40$ and $1044 \pm 29 \Omega /$ sq, respectively. The observed behavior can be explained by the formation of multilayer and domains structure of the graphene layer due to different nucleation densities as reported in our previous article [46]. Recently, Chamoli et al. and his co-workers have developed low defect density graphene nanosheets (GNs) have via chemical reduction of exfoliated graphite (EG) in the presence of a green reducing agent for transparent conductive film $[47,48]$. They have achieved the transmittance value of $\sim 71.5 \%$ and sheet resistance of $\sim 1 \mathrm{~K} \Omega / \mathrm{sq}$. Comparing both the results, we have achieved a very high values of transmittance and moderate sheet resistance values. This shows that the Cu pretreatment helps to remove the native copper oxide and impurity particles on the Cu foil.

Finally, we have fabricated and characterized the graphene/n-Si schottky junction diode using graphene grown on $\mathrm{HCl}$ pretreated $\mathrm{Cu}$ foil because it showed excellent transmittance and optimum sheet resistance values. Schottky diodes are formed by making contact between the metal and semiconductor [49]. These diodes are nowadays providing a platform for a variety of optoelectronic devices such as solar cells, photodetectors, RF attenuators, chemical sensors, etc. [51-53]. Graphene/semiconductor schottky junction diodes are widely studied because the Fermi-level of the graphene can be varied with the applied potential $[53,54]$. Figure 8 (a) shows a schematic diagram of the fabricated graphene/metal device on $\mathrm{SiO}_{2} / \mathrm{Si}$ substrate with $\mathrm{Ag}$ as contact electrode. The $J-V$ characteristic of the as chosen $\mathrm{HCl}$ pre-treated $\mathrm{Cu}$ foil derived camphor based graphene is investigated. The device showed the rectifying behavior which can be seen from the current-voltage characteristics. The pretreated $\mathrm{Gr} / \mathrm{n}$-Si shows a dramatic change in the rectification behavior of $5.5 \mu \mathrm{A} / \mathrm{cm}^{2}$ at $-0.5 \mathrm{~V}$, which is found to be more than the as-grown $\mathrm{Gr} / \mathrm{n}-\mathrm{Si}$ device with $\mathrm{J}_{\mathrm{SC}} \leq 5 \mu \mathrm{A} / \mathrm{cm}^{2}$ reported in our previous article [55]. Table 1 demonstrates the performances parameters of various devices developed under different conditions to deliver a prominent currant different desired bias voltages. It is observed that, at $\mathrm{V}_{\mathrm{b}}=0 \mathrm{~V}$, the $\mathrm{J}_{\mathrm{SC}}$ values are not as high as it is expected for all the devices. Our present work also shows a similar results when the $\mathrm{Cu}$ foils are pretreated with $\mathrm{HCl}$. The Ag electrodes make an ohmic contact to the graphene, while the $\mathrm{n}-\mathrm{Si} /$ graphene interface forms a Schottky barrier. The typical dark and light $\mathrm{J}-\mathrm{V}$ characteristic of $\mathrm{HCl}$ treated graphene/n-Si heterojunction device is shown in Fig. 8 (b). The mono/bi layer graphene on the top of n-Si demonstrates the photodiode like behavior for the fabricated device. The increase in the photocurrent under reverse bias condition has been observed under light due to large number of accessible states of collection of holes in Si that inject into the graphene [54]. In addition, the inset shows the light chopped J$\mathrm{V}$ curve under reverse bias condition for $\mathrm{V}_{\text {bias }}$ typically from -5 to $0 \mathrm{~V}$. The excellent increment in the photocurrent with increase in the bias voltage has been observed for a typical photodiode with a very high sensitivity. Thus, the $\mathrm{J}-\mathrm{V}$ characteristic and the rectifying nature confirms the formation of a Schottky junction at the graphene/n-Si. 
Table 1

Device performances of MLG/Si based Schottky junction devices

\begin{tabular}{|lll|}
\hline Device structure & Current Density $\left(\mathrm{J}_{\mathrm{SC}}\right)$ at 0 V & References \\
\hline $\mathrm{Gr} / \mathrm{p}-\mathrm{Si}$ & $<0.5 \mathrm{~mA} / \mathrm{cm}^{2}$ & 56 \\
\hline Pristine $\mathrm{Gr} / \mathrm{n}$-Si & $4 \mu \mathrm{A} / \mathrm{cm}^{2}$ & 57 \\
\hline $\mathrm{HCl}$ treated Cu foil Gr/n-Si & $1.2 \mu \mathrm{A} / \mathrm{cm}^{2}$ & Our work \\
\hline $\mathrm{n}$-Si pristine MLG & $3 \mu \mathrm{A} / \mathrm{cm}^{2}$ & 58 \\
\hline
\end{tabular}

\section{Conclusion}

In summary, we have investigated the different pretreatment conditions of Cu foils such as acetone, AA, $\mathrm{HCl}$ and $\mathrm{HNO}_{3}$ on the graphene growth and final properties of graphene grown from APCVD technique using camphor as a solid carbon precursor. Our results suggested that the $\mathrm{HCl}$ pretreatment improved the surface properties of the $\mathrm{Cu}$ foil which reduces the graphene nucleation density and thus the largedomain can be obtained. SEM and AFM studies revealed that the $\mathrm{Cu}$ foil pretreated with $\mathrm{HCl}$ shown better smoothness when compared to other pretreatment conditions. It was found that the reduction in the surface impurity particles and RMS after $\mathrm{HCl}$ pretreatment. The oxidation study showed the uniform coverage of the graphene on the $\mathrm{Cu}$ foil obtained from $\mathrm{HCl}$ pretreatment. The graphene transferred on to the $\mathrm{SiO}_{2} / \mathrm{Si}$ studied using Raman spectroscopy showed that $\mathrm{HCl}$ pretreated $\mathrm{Cu}$ foil exhibited $\mathrm{I}_{2 \mathrm{D}} / \mathrm{I}_{\mathrm{G}}$ ratio of 1.35 and $I_{D} / I_{G}$ ratio of 0.05 which showed the high quality of graphene grown from camphor. The optical transmittance of $~ 91.5 \%$ and sheet resistance of $861 \pm 40 \mathrm{ohm} / \mathrm{sq}$ were achieved using $\mathrm{HCl}$ pretreated $\mathrm{Cu}$ foil. Further, we transferred the graphene on to the $\mathrm{SiO}_{2} / \mathrm{Si}$ and investigated the graphene/n-Si schottky diode characteristics.

\section{Declarations}

\section{Conflicts of Interest}

There are no conflicts of interest to declare.

\section{Acknowledgements}

One of the authors (Dr. R. Narasimman) would like to acknowledge the DST-SERB, New Delhi, India for the award of SERB National Post-Doctoral Fellowship (File Number: PDF/2017/003063 dated: 11/07/2017). The authors are acknowledging IIT, Gandhinagar for AFM facility.

\section{References}


[1] Novoselov KS, Geim AK, Morozov SV, Jiang D, Zhang Y, Dubonos SV, et al. ElectricField Effect in Atomically Thin Carbon Films. Science. 2004;306(5696):666.

[2] Bunch JS, van der Zande AM, Verbridge SS, Frank IW, Tanenbaum DM, Parpia JM, et al. Electromechanical Resonators from Graphene Sheets. Science. 2007;315(5811):490.

[3] Wu J, Pisula W, Müllen K. Graphenes as Potential Material for Electronics. ChemicalReviews. 2007;107(3):718-47.

[4] Lee C, Wei X, Kysar JW, Hone J. Measurement of the Elastic Properties and IntrinsicStrength of Monolayer Graphene. Science. 2008;321(5887):385.

[5] Balandin AA. Thermal properties of graphene and nanostructured carbon materials. Nature Materials. 2011;10:569.

[6] Liu M, Yin X, Ulin-Avila E, Geng B, Zentgraf T, Ju L, et al. A graphene-based broadband optical modulator. Nature. 2011;474:64.

[7] Li X, Zhu Y, Cai W, Borysiak M, Han B, Chen D, et al. Transfer of Large-AreaGraphene Films for High-Performance Transparent Conductive Electrodes. Nano Letters.2009;9(12):4359-63.

[8] Xia F, Mueller T, Lin Y-m, Valdes-Garcia A, Avouris P. Ultrafast graphene photodetector. Nature Nanotechnology. 2009;4:839.

[9] Kim KS, Zhao Y, Jang H, Lee SY, Kim JM, Kim KS, et al. Large-scale pattern growth of graphene films for stretchable transparent electrodes. Nature. 2009;457:706.

[10] Li X, Cai W, An J, Kim S, Nah J, Yang D, et al. Large-Area Synthesis of High-Quality and Uniform Graphene Films on Copper Foils. Science. 2009;324(5932):1312.

[11] Emtsev KV, Speck F, Seyller T, Ley L, Riley JD. Interaction, growth, and ordering of epitaxial graphene on $\mathrm{SiC}\{0001\}$ surfaces: A comparative photoelectron spectroscopy study. Physical Review B. 2008;77(15):155303.

[12] Eda G, Fanchini G, Chhowalla M. Large-area ultrathin films of reduced graphene oxide as a transparent and flexible electronic material. Nature Nanotechnology. 2008;3:270.

[13] Becerril HA, Mao J, Liu Z, Stoltenberg RM, Bao Z, Chen Y. Evaluation of Solution- Processed Reduced Graphene Oxide Films as Transparent Conductors. ACS Nano.2008;2(3):463-70.

[14] Gao L, Ren W, Xu H, Jin L, Wang Z, Ma T, et al. Repeated growth and bubbling transfer of graphene with millimetre-size single-crystal grains using platinum. Nature Communications.2012;3:699.

[15] Alpha TND, Johann C, Tim NP, Carsten B, Thomas M. Structure of epitaxial graphene on $\operatorname{Ir}(111)$. New Journal of Physics. 2008;10(4):043033. 
[16] Qi M, Ren Z, Jiao Y, Zhou Y, Xu X, Li W, et al. Hydrogen Kinetics on Scalable Graphene Growth by Atmospheric Pressure Chemical Vapor Deposition with Acetylene. The Journal of Physical Chemistry C. 2013;117(27):14348-53.

[17] Zhao L, Rim KT, Zhou H, He R, Heinz TF, Pinczuk A, et al. Influence of copper crystal surface on the CVD growth of large area monolayer graphene. Solid State Communications.2011;151(7):509-13.

[18] Dong X, Wang P, Fang W, Su C-Y, Chen Y-H, Li L-J, et al. Growth of large-sized graphene thin-films by liquid precursor-based chemical vapor deposition under atmospheric pressure. Carbon. 2011;49(11):3672-8.

[19] Guermoune A, Chari T, Popescu F, Sabri SS, Guillemette J, Skulason HS, et al. Chemical vapor deposition synthesis of graphene on copper with methanol, ethanol, and propanol precursors. Carbon. 2011;49(13):4204-10.

[20] Kalita G, Masahiro M, Uchida H, Wakita K, Umeno M. Few layers of graphene as transparent electrode from botanical derivative camphor. Materials Letters. 2010;64(20):2180-3.

[21] Kalita G, Wakita K, Umeno M. Monolayer graphene from a green solid precursor. Physica E: Lowdimensional Systems and Nanostructures. 2011;43(8):1490-3.

[22] Ravani F, Papagelis K, Dracopoulos V, Parthenios J, Dassios KG, Siokou A, et al. Graphene production by dissociation of camphor molecules on nickel substrate. Thin Solid Films. 2013;527:31-7.

[23] Sharma S, Kalita G, Ayhan ME, Wakita K, Umeno M, Tanemura M. Synthesis of hexagonal graphene on polycrystalline $\mathrm{Cu}$ foil from solid camphor by atmospheric pressure chemical vapor deposition. Journal of Materials Science. 2013;48(20):7036-41.

[24] Sharma S, Kalita G, Hirano R, Hayashi Y, Tanemura M. Influence of gas composition on the formation of graphene domain synthesized from camphor. Materials Letters. 2013;93:258-62.

[25] Kobayashi T, Bando M, Kimura N, Shimizu K, Kadono K, Umezu N, et al. Production of a 100-m-long high-quality graphene transparent conductive film by roll-to-roll chemical vapor deposition and transfer process. Applied Physics Letters. 2013;102(2):023112.

[26] Bae S, Kim H, Lee Y, Xu X, Park J-S, Zheng Y, et al. Roll-to-roll production of 30-inch graphene films for transparent electrodes. Nature Nanotechnology. 2010;5:574.

[27] López GA, Mittemeijer EJ. The solubility of C in solid Cu. Scripta Materialia.2004;51(1):1-5.

[28] Li X, Cai W, Colombo L, Ruoff RS. Evolution of Graphene Growth on Ni and Cu byCarbon Isotope Labeling. Nano Letters. 2009;9(12):4268-72. 
[29] Yu Q, Jauregui LA, Wu W, Colby R, Tian J, Su Z, et al. Control and characterization of individual grains and grain boundaries in graphene grown by chemical vapour deposition. Nature Materials. 2011;10:443.

[30] Mattevi C, Kim H, Chhowalla M. A review of chemical vapour deposition of graphene on copper. Journal of Materials Chemistry. 2011;21(10):3324-34.

[31] Reckinger N, Felten A, Santos CN, Hackens B, Colomer J-F. The influence of residual oxidizing impurities on the synthesis of graphene by atmospheric pressure chemical vapor deposition. Carbon. 2013;63:84-91.

[32] Soo Min K, Allen H, Yi-Hsien L, Mildred D, Tomás P, Ki Kang K, et al. The effect of copper pre-cleaning on graphene synthesis. Nanotechnology. 2013;24(36):365602.

[33] Kim M-S, Woo J-M, Geum D-M, Rani JR, Jang J-H. Effect of copper surface pre- treatment on the properties of CVD grown graphene. AIP Advances. 2014;4(12):127107.

[34] Gnanaprakasa TJ, Gu Y, Eddy SK, Han Z, Beck WJ, Muralidharan K, et al. The role of copper pretreatment on the morphology of graphene grown by chemical vapor deposition. Microelectronic Engineering. 2015;131:1-7.

[35] Ibrahim A, Nadhreen G, Akhtar S, Kafiah FM, Laoui T. Study of the impact of chemical etching on Cu surface morphology, graphene growth and transfer on SiO2/Si substrate. Carbon.2017;123:402-14.

[36] Murdock AT, van Engers CD, Britton J, Babenko V, Meysami SS, Bishop H, et al. Targeted removal of copper foil surface impurities for improved synthesis of CVD graphene. Carbon. 2017;122:207-16.

[37] Senyildiz D, Ogurtani OT, Cambaz Buke G. The effects of acid pretreatment and surface stresses on the evolution of impurity clusters and graphene formation on $\mathrm{Cu}$ foil. Applied Surface Science. 2017;425:873-8.

[38] Huet B, Raskin J-P. Role of Cu foil in-situ annealing in controlling the size and thickness of CVD graphene domains. Carbon. 2018;129:270-80.

[39] Vlassiouk I, Fulvio P, Meyer H, Lavrik N, Dai S, Datskos P, et al. Large scale atmospheric pressure chemical vapor deposition of graphene. Carbon. 2013;54:58-67.

[40] Lee D, Kwon GD, Kim JH, Moyen E, Lee YH, Baik S, et al. Significant enhancement of the electrical transport properties of graphene films by controlling the surface roughness of $\mathrm{Cu}$ foils before and during chemical vapor deposition. Nanoscale. 2014;6(21):12943-51.

[41] Dhingra S, Hsu J-F, Vlassiouk I, D'Urso B. Chemical vapor deposition of graphene onlarge-domain ultra-flat copper. Carbon. 2014;69:188-93. 
[42] Chavez KL, Hess DW. A Novel Method of Etching Copper Oxide Using Acetic Acid. Journal of The Electrochemical Society. 2001;148(11):G640-G3.

[43] Luo Z, Lu Y, Singer DW, Berck ME, Somers LA, Goldsmith BR, et al. Effect of Substrate Roughness and Feedstock Concentration on Growth of Wafer-Scale Graphene at Atmospheric Pressure. Chemistry of Materials. 2011;23(6):1441-7.

[44] Malard LM, Pimenta MA, Dresselhaus G, Dresselhaus MS. Raman spectroscopy in graphene. Physics Reports. 2009;473(5):51-87.

[45] Ferrari AC, Basko DM. Raman spectroscopy as a versatile tool for studying the properties of graphene. Nature Nanotechnology. 2013;8:235.

[46] Chaliyawala H, Patel R, Narasimman R, Ray A and Mukhopadhyay. Controlled Island Formation of Large-Area Graphene Sheets by Atmospheric Chemical Vapor Deposition: Role of Natural Camphor. ACS Omega. 2019: 4: 5: 8758-8766.

[47] Chamoli P, Das M.K., Kar K.K., Urea-assisted low temperature green synthesis of graphene nanosheets for transparent conducting film, Journal of Physics and Chemistry of Solids:2017: doi: 10.1016/j.jpcs.2017.10.001.

[48] Chamoli P, Das M.K., Kar K.K. Green synthesis of silver-graphene nanocomposite-based transparent conducting film, Physica E. 2017; 90: 76-84.

[49] Di Bartolomeo A. Graphene Schottky diodes: An experimental review of the rectifying graphene/semiconductor heterojunction. Physics Reports. 2016;606:1-58.

[50] Kumar M, Patel M, Kim H-S, Kim J, Yi J. High-Speed, Self-Biased Broadband Photodetector-Based on a Solution-Processed Ag Nanowire/Si Schottky Junction. ACS Applied Materials \& Interfaces. 2017;9(44):38824-31.

[51] Kobayashi M, Kinoshita A, Saraswat K, Wong HSP, Nishi Y. Fermi level depinning in metal/Ge Schottky junction for metal source/drain Ge metal-oxide-semiconductor field-effect- transistor application. Journal of Applied Physics. 2009;105(2):023702.

[52] Fute Z, Tao S, Baoquan S. Conjugated polymer-silicon nanowire array hybrid Schottky diode for solar cell application. Nanotechnology. 2012;23(19):194006.

[53] Chen C-C, Aykol M, Chang C-C, Levi AFJ, Cronin SB. Graphene-Silicon Schottky Diodes. Nano Letters. 2011;11(5):1863-7.

[54] Sinha D, Lee JU. I deal Graphene/Silicon Schottky Junction Diodes. Nano Letters. 2014;14(8):4660-4. 
[55] Chaliyawala H, Aggarwal N, Purohit Z, Patel R, Gupta G, Jaffre A, Le Gall S, Ray A and Mukhopadhyay I Role of nanowire length on the performance of a self-driven NIR photodetector based on mono/bi-layer graphene (camphor)/Si-nanowire Schottky junction. 2020: 31: 225208.

[56] Mohammed M, Li Z, Cui J and Chen T. Junction investigation of graphene/silicon Schottky diodes. Nanoscale Res. Lett. 2012: 7:302

[57] Xiang D, Han C, Hu Z, Lei B, Liu Y, Wang L, Hu W P and Chen W. Surface Transfer Doping-Induced, High-Performance Graphene/Silicon Schottky Junction-Based, Self- Powered Photodetector. Small 2015: vol. 11 No. $37,4829-4836$.

[58] Chao Xie, Peng Lv, Biao Nie, Jiansheng Jie, Xiwei Zhang, Zhi Wang, Peng Jiang, Zhizhong Hu, Linbao Luo, Zhifeng, Zhu, Li Wang, and Chunyan Wu. Monolayer graphene film/silicon nanowire array Schottky junction solar cells. Appl. Phys. Lett. 2011: 99, 133113.

\section{Figures}



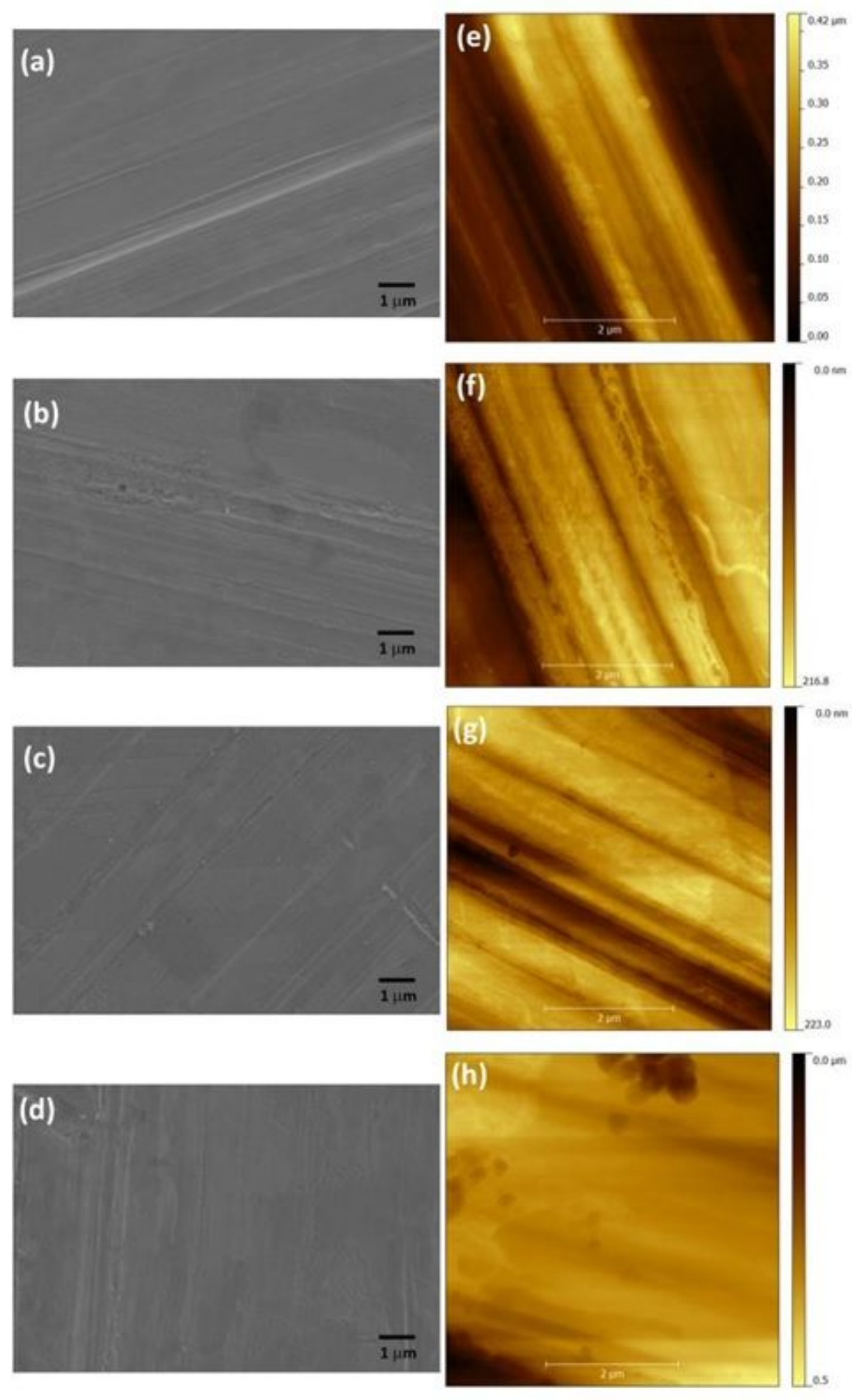

\section{Figure 1}

SEM and AFM micrographs of pretreated Cu foils at different conditions (a-e) Acetone, (b-f) Acetic acid (10 mins), (c-g) HCl (2:1 HCl:H2O, 10 mins) and (d-h) HNO3 (5 wt.\%, $30 \mathrm{sec})$ 

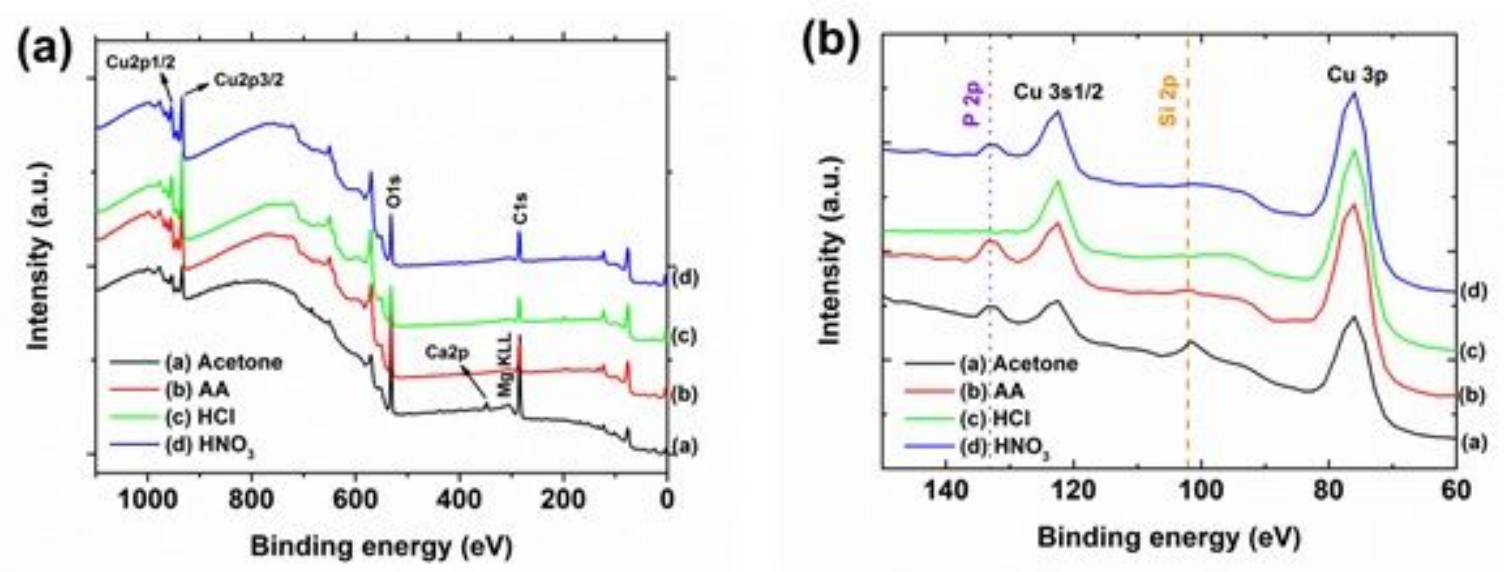

Figure 2

(a) XPS survey spectra of the pretreated Cu foils at different conditions (Acetone, Acetic acid (10 mins), $\mathrm{HCl}(2: 1 \mathrm{HCl}: \mathrm{H} 2 \mathrm{O}, 10 \mathrm{mins})$ and $\mathrm{HNO} 3(5 \mathrm{wt} . \%, 30 \mathrm{sec})$ and (b) XPS survey spectra of the magnified portion in the range of 150 to $60 \mathrm{eV}$.
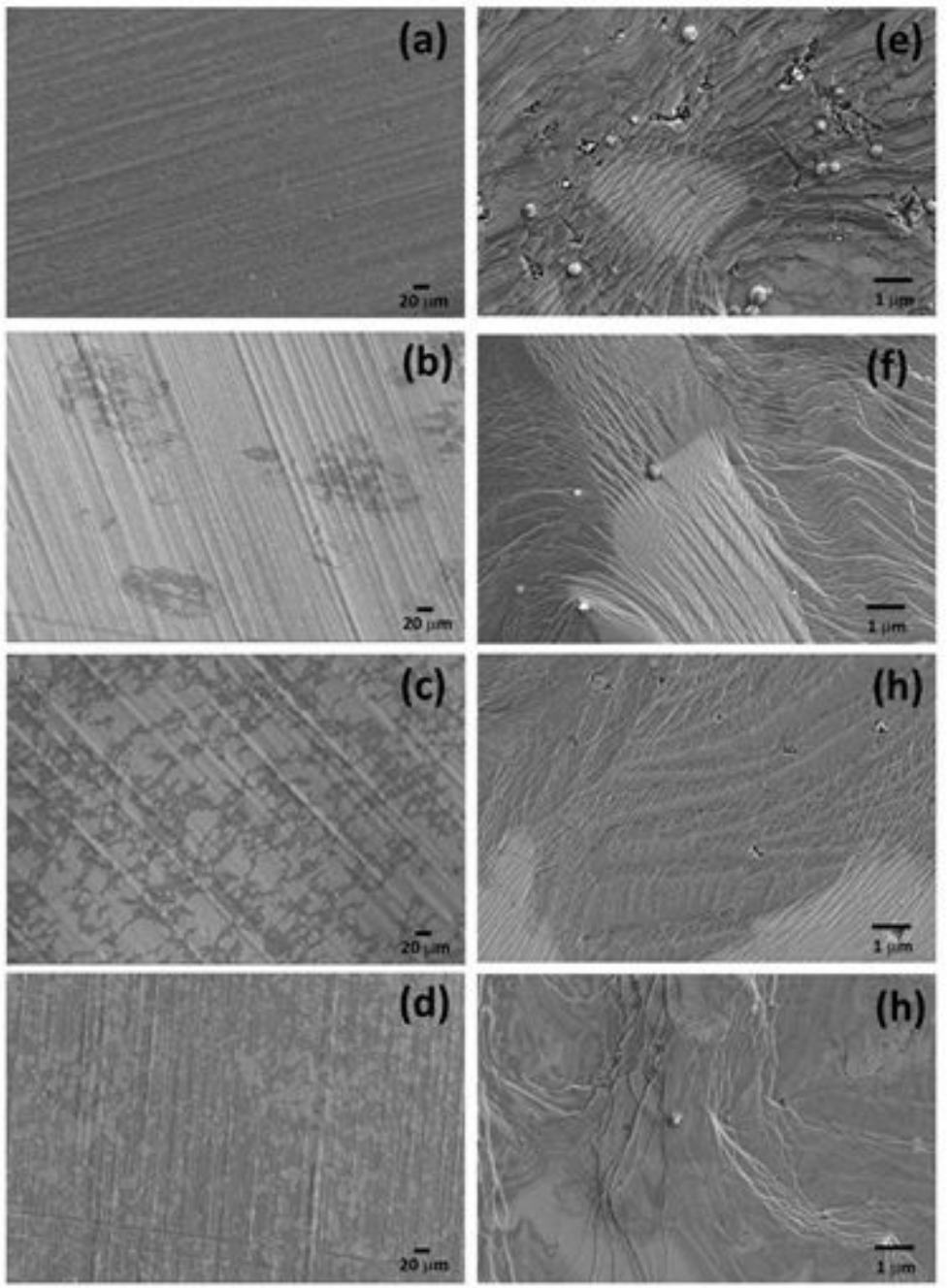

Figure 3 
SEM micrographs of graphene grown on Cu foils which pretreated at different conditions as follows: $(a, e)$ Acetone, (b,f) Acetic acid (10 mins), (c,g) HCl (2:1 HCl:H2O, 10 min), and (d,h) HNO3 (5 wt.\%, 30 sec)
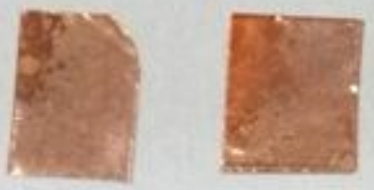

Acetone

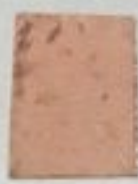

$\mathrm{HCl}$

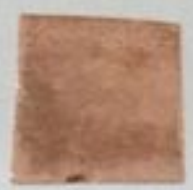

$\mathrm{HNO}_{3}$

\section{Figure 4}

Digital photograph of the graphene grown on Cu foils which pretreated at different conditions after oxidation treatment at $2000 \mathrm{C}$ for 10 mins in air atmosphere
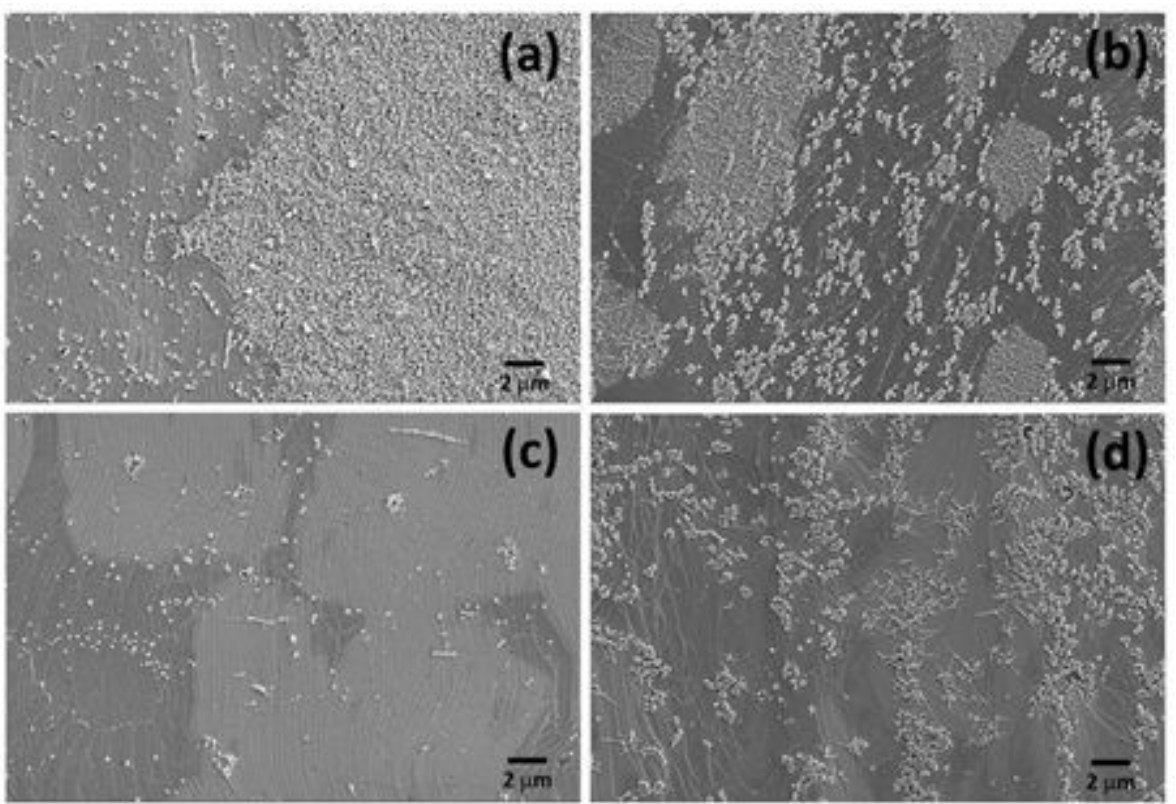

\section{Figure 5}

SEM images of oxidized graphene grown $\mathrm{Cu}$ foils which pretreated at different conditions after oxidation treatment at $200 \mathrm{oC}$ for 10 mins in air atmosphere [(a) Acetone (b) Acetic acid (10 mins) (c) $\mathrm{HCl}(2: 1$ $\mathrm{HCl}: \mathrm{H} 2 \mathrm{O}, 10$ mins) and (d) HNO3 (5 wt.\%, $30 \mathrm{sec}$ )] 

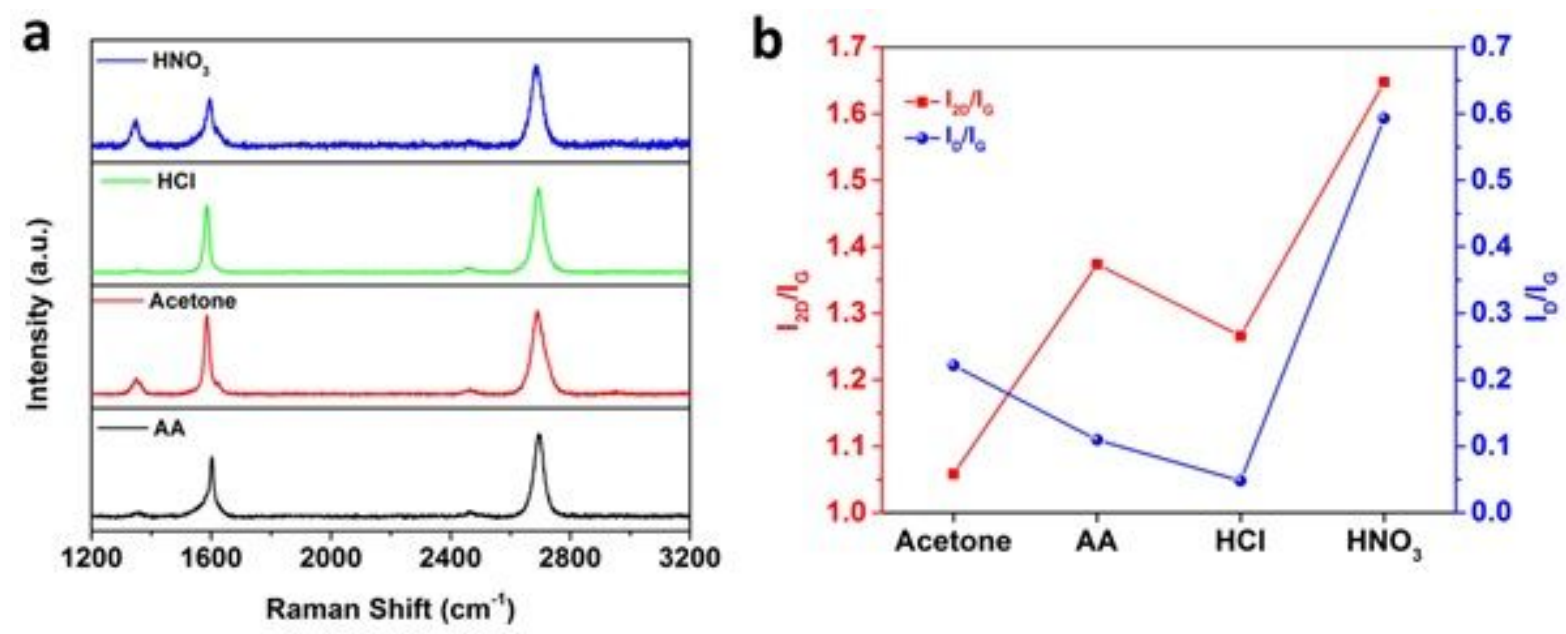

Figure 6

(a) Raman spectra and (b) I2D/IG and ID/IG ratios of the graphene grown on pretreated Cu foils on $\mathrm{SiO} 2 / \mathrm{Si}$ substrate with different pretreatment conditions

(a)

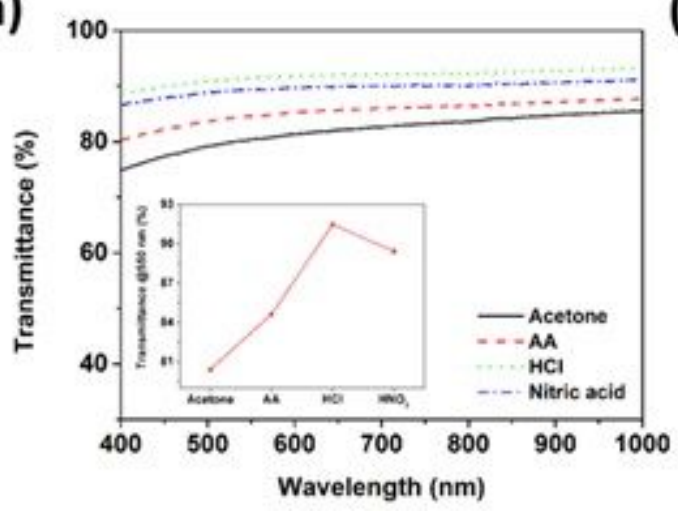

(b)

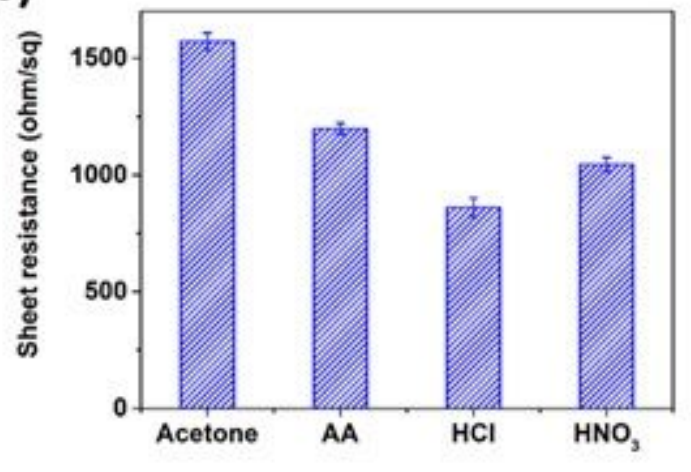

Figure 7

(a) UV-visible transmittance spectra and (b) sheet resistance of graphene grown on pretreated Cu foils transferred on to glass substrate.

(a)

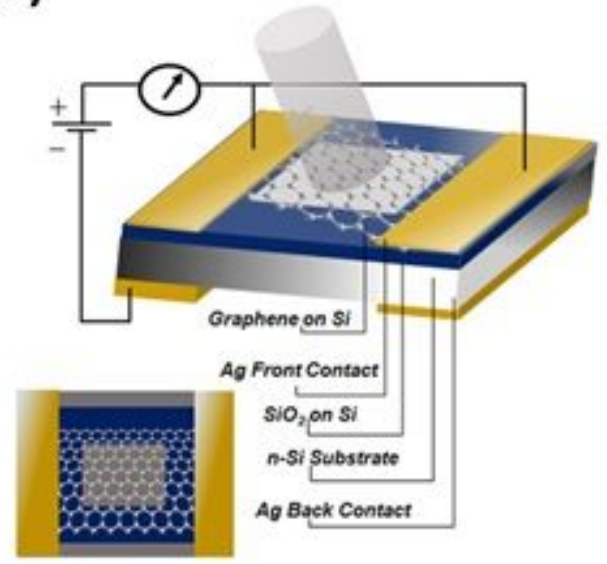

(b)

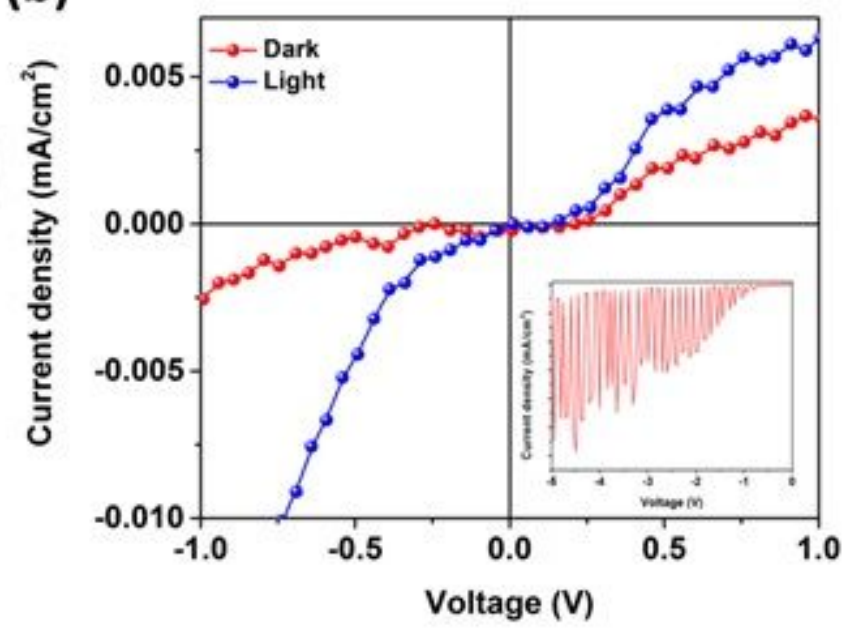




\section{Figure 8}

(a) Schematic photograph of the graphene/n-Si Schottky junction photodiode (graphene grown on the pretreated $\mathrm{Cu}$ foil with $\mathrm{HCl}$ ) and (b) J-V characteristics of the photodiode in dark and light conditions (100 $\mathrm{mW} / \mathrm{cm} 2$ ). Inset in (b) shows the light chopped J-V curve under reverse bias condition for Vbias typically from -5 to $0 \mathrm{~V}$. 